\title{
Letter to the editor: Post-traumatic stress in head and neck cancer survivors and their partners
}

\author{
Hang Yang ${ }^{1}$ \\ Received: 20 August 2018 / Accepted: 11 October 2018 / Published online: 17 October 2018 \\ (C) Springer-Verlag GmbH Germany, part of Springer Nature 2018
}

To the editor,

In a previous issue of Supportive Care in Cancer, the article from Elisavet Moschopoulou et al. [1] has indicated that symptoms of cancer-related post-traumatic stress disorder (PTSD) exist for many years and also affect caregivers in some head and neck cancer (HNC) patients. The authors first concluded the psychological states of HNC survivors for clinicians. As a result, some questions or ideas on its causes and solutions will follow.

As treatment for HNC survivors has been clinically meaningful in prevention of its progression, does it have any effect on patients' psychological states that will change after receiving effective therapy? So how to change, worse or better, may be a way to assess the effect of treatment of HNC on psychological states. For example, receiving treatment of HNC rather than specific psychotherapy will be beneficial for patients' psychological states, if they are mentally healthier after specific treatment of HNC than before. The decreasing ratio of patients first identified as cancer-related PTSD after treatment to the patients with anxiety or depression all the time also hints the dual improving effect on disease itself and psychological stages related with HNC. In addition, more research has suggested the high rate of PTSD in intensive care unit (ICU), and the proactive communication was showed to decrease the rate
[2]. Therefore, is there structured and proactive communication with quality for patients and caregivers from attending doctors or their psychologists when the HNC treatment can not relieve or even induce more serious anxious or depressed state? After all, one of the roles of clinical research can act as clinical evidence and help clinicians make more reasonable assumption of diseases including evolution and prognosis, which is also a part of high-quality conversation. For example, I have known about the potential possibility of cancer-related PTSD in HNC patients and their caregivers after reading the article, so some assessment of initial psychological states and prospective conversation will be carried out when treating HNC patients.

\section{References}

1. Moschopoulou E, Hutchison I, Bhui K, Korszun A (2018) Posttraumatic stress in head and neck cancer survivors and their partners. Support Care Cancer 26(9):3003-3011

2. Zimmerli M, Tisljar K, Balestra GM, Langewitz W, Marsch S, Hunziker S (2014) Prevalence and risk factors for post-traumatic stress disorder in relatives of out-of-hospital cardiac arrest patients. Resuscitation 85(6):801-808
Hang Yang

15841123892@163.com

1 Department of Emergency Medicine, Second Affiliated Hospital, School of Medicine and Institute of Emergency Medicine, Zhejiang University, Hangzhou, China 